\title{
PROCEDURES USED AT STATEVILLE PENITENTIARY FOR THE TESTING OF POTENTIAL ANTIMALARIAL AGENTS ${ }^{1}$
}

\author{
By ALF S. ALVING, BRANCH CRAIGE, JR.,2 THEODORE N. PULlMAN, ${ }^{2}$ \\ C. MERRILL WHORTON,2 RALPH JONES, JR.,2 \\ AND LILLIAN EICHELBERGER \\ (From the Malarial Research Unit, Department of Medicine, University of Chicago)
}

(Received for publication December 23, 1946)

\section{INTRODUCTION}

The need for normal human subjects to use in appraising the activity of antimalarial drugs led, in 1944, to the establishment of a clinical research unit at the Illinois State Penitentiary, Stateville, Illinois. Through an arrangement with the Department of Public Safety, ${ }^{3}$ one floor of the prison hospital and a portion of a second floor were placed at the disposal of the Malaria Research Project. Approximately 500 inmates volunteered to act as subjects.

The studies were designed primarily to yield in-

1 This investigation was carried out under contract, recommended by the Committee on Medical Research, between the Office of Scientific Research and Development and the University of Chicago. The studies were planned in cooperation with the Panel on Clinical Testing of Antimalarials of the Board for the Coordination of Malarial Studies. This work was further aided by the participation of Army Medical officers assigned to the project by the Surgeon General, U. S. Army.

Through a cooperative arrangement between Professor Clay G. Huff and Dr. Frederick Coulston, Department of Bacteriology and Parasitology, and the Malarial Research Unit, Department of Medicine, the former group bred Anopheles quadrimaculatus mosquitoes, supervised their infection and the inoculation of volunteers, and determined the intensity of infection in the salivary glands of the mosquitoes. The latter group assumed the responsibility for clinical care of patients studied by both groups.

The authors express their thanks to the Malaria Study Section of the National Institute of Health for editorialassistance and for arrangements in regard to the publication of this paper. They are also grateful to the Abbott Laboratories, E. I. du Pont de Nemours and Company, Inc., E. R. Squibb and Sons, Eli Lilly and Company, Sharp and Dohme, Inc., and Wyeth, Inc., for contributing toward the publication costs.

2 Formerly Captain, M.C., A.U.S.

3 The authors wish to acknowledge the cooperation of the following officials in the State of Illinois who made these arrangements possible: Dwight $H$. Green, Governor, T. P. Sullivan, Director of Public Safety, and Joseph E. Ragen, Warden of Illinois State Penitentiary at JolietStateville. formation concerning the effect of potential antimalarial agents upon the relapse rate of sporozoiteinduced vivax malaria. The observations also furnished information on the prophylactic and suppressive effects of the drugs tested, as well as data on their toxicology and pharmacology in man. The Chesson strain of malaria was selected for study because its short latent period between attacks (1) made feasible the rapid accumulation of information. An abundance of normal volunteers, in the younger age groups, living under standard conditions of diet and daily routine, made controlled clinical testing of antimalarial drugs possible. The institution being in a non-endemic area, accidental reinoculation was not a problem.

\section{PROPHYLACTIC TESTS}

The subjects were white, male inmate volunteers in good physical health. They were acquainted beforehand with the nature of the disease and the general plan of the study. Nearly all men were in the age group of 21 to 40 . Only those whose stay in the institution was ascertained to be 18 months or longer were selected. Follow-up observations could be made in nearly 100 per cent of the subjects. Volunteers who had lived in known malarious areas, or who gave a history suggestive of previous malarial infection, or who belonged to one of the colored races, were excluded in order to minimize the factors of acquired or natural immunity.

A medical history was taken and physical examinations were made on all candidates. In addition, the following procedures were routine: complete blood count, urinalysis, urinary urobilinogen concentration, phenolsulfonephthalein excretion, cephalin-cholesterol flocculation, serum bilirubin, blood nonprotein nitrogen, blood Kahn, blood typing, chest x-ray, electrocardiogram, and, where indicated, erythrocyte sedimentation rate. 
Malaria parasite. The Chesson strain of $P$. vivax (2) was isolated from a military patient who presumably had acquired his infection in $\mathrm{New}$ Guinea and was under treatment at the Harmon General Hospital, Longview, Texas (3). This strain is characterized by a high relapse rate when treated with noncurative drugs such as quinine and quinacrine, by a short period of latency between successive attacks, and by almost complete absence of delayed primary attacks $(1,4)$. The strain was maintained in psychotic patients at the Manteno State Hospital, Manteno, Illinois," chiefly by blood inoculations from donors who had manifested high gametocyte densities during trophozoite-induced infections. Not infrequently, however, donors were Stateville volunteers, whose malaria had been sporozoite-induced.

Transmission. Mosquito infection was carried out under the supervision of Drs. Clay G. Huff and Frederick Coulston of the University of Chicago, Department of Bacteriology and Parasitology. Anopheles quadrimaculatus mosquitoes were permitted to feed on patients whose gametocyte densities were such as to insure a reasonably high incidence of infection in the biting mosquitoes. They were then incubated for suitable intervals in the mosquito insectory at the University of Chicago.

Inoculation of volunteers. The technique of initiating sporozoite-induced infections was a modification of that used by Coatney and his coworkers (5) at the Federal Penitentiary at Atlanta, Ga. Mosquito lots were used in which the incidence of infection was high, as determined by random sampling. Each patient received the bites of ten infected mosquitoes. After feeding, the mosquitoes were dissected and a microscopic examination made of the salivary glands to determine the degree of infection. Each mosquito was kept in an individual plastic cylindrical cage $4.5 \mathrm{~cm}$. in height, the ends of which were covered by coarse mesh. In most instances, the mosquito was per-

\footnotetext{
4 The facilities of the Manteno State Hospital were made available for these studies through the cooperation of Mr. Rodney H. Brandon and General Cassius Poust, former and present Directors of Public Welfare; Drs. Conrad Sommer and George A. Wiltrakis, former and present Deputy Directors, Department of Public Welfare, and Drs. Edward Ross and Walter H. Baer, former and present Superintendents of Manteno State Hospital.
}

mitted to bite two or three subjects consecutively, the first feedings being interrupted before blood was visible in the abdomen of the mosquito. The last feeding was permitted to proceed until the mosquito stopped spontaneously or became engorged with blood. When a mosquito refused further feeding after it had already bitten one or two patients in a group, a substituted mosquito was allowed to complete the inoculation of the given group of men. Similarly, when mosquitoes were found on dissection to be uninfected, substitute mosquitoes were fed on the same subject.

In the multiple-bite technique, which was the usual procedure for inoculation, each man received, as nearly as possible, the same number of first, second, and third bites. Men receiving no drug and, therefore, serving as control subjects, were inoculated at the same time as those receiving drug for prophylactic testing. The group bitten by the same mosquitoes included at least one control subject.

The technique of infecting the subjects on inoculation day varied, and assumed one of several basic patterns, as follows:

1. Most commonly the patients were inoculated in groups of three.

2. The men were occasionally inoculated in pairs, each pair receiving the bites of the same ten infected mosquitoes.

3. In exceptional instances each subject was bitten separately by ten infected mosquitoes.

Administration of drugs. Three to five volunteers were employed for prophylactic tests. In the standard prophylactic procedure, the drug was administered on the day before inoculation, the day of inoculation, and for the following six days. Drugs were administered in gelatin capsules at four-hour intervals to obtain fairly constant concentration in the body fluids.

It was necessary to take elaborate precautions in order to avoid errors in dosage. At the start of observations, the exact number of capsules required for each patient's entire course of treatment was placed in individual bottles. An individual treatment sheet was also prepared. This sheet contained the following information: the patient's name, the code number of the drug, the 
detailed schedule of drug administration for the entire course of therapy, and the number of capsules that should remain in the bottle at the end of each day. The bottle and complete treatment schedules were given to the nurses. The capsules remaining in each bottle were counted daily and checked with the original schedule. At the time of the daily tally, enough capsules for the ensuing 24-hour period were removed from the original bottle and each dose was placed in a single small vial, on the label of which had been noted the name of the patient and the hour for administration. When an individual dose was administered, the nurse signed the treatment schedule opposite the hour for which the dose had been scheduled.

Observations. Thick films of the peripheral blood were taken daily after the eighth day following inoculation. When fever appeared, the films were read, and, if positive, previous films were examined. The films were of capillary blood and were prepared and stained according to the method of Earle and Perez (6). Sufficient area of a film was inspected microscopically to insure counting of all the parasites contained in $0.1 \mathrm{c} . \mathrm{mm}$. of whole blood. The count was then referred to 1.0 c. $\mathrm{mm}$.

The oral temperature and pulse were recorded when the thick films were made. Subjects were admitted to the hospital when fever or symptoms made ambulatory status inadvisable.

During the periods of hopitalization, observations were made as follows:

1. Rectal temperature, pulse, and respirations every four hours. When the rectal temperature was above $101^{\circ} \mathrm{F}$., these observations were made every 30 minutes.

2. Blood pressure every day.

3. Total daily fluid intake and urine volume.

4. Daily urinalysis.

5. Determination of plasma drug concentrations every day or on alternate days; more frequently if special information was desired, as in the case of rapidly excreted or rapidly degraded drugs.

6. Special tests necessary to detect and study toxicity.

\section{CURATIVE TESTS}

Subjects. The volunteers were selected and inoculated as described for the prophylactic studies. In a few cases inoculation was achieved by the injection, intravenously, subcutaneously, or into lymph nodes, of a suspension of infected mosquito salivary glands. Many subjects had served in tests in which prophylaxis had failed, or had belonged to the control group. Only patients having primary attacks, first and second relapses, were used as subjects in tests where it was desirable to minimize the effects of immunity. Successive clinical attacks in the same individual were used to study the therapeutic properties of different drugs. Later observations (1), however, indicated that attacks in patients whose preceding latent period was prolonged, presented a milder therapeutic challenge to a test drug. Consequently, in the later curative trials whenever the amount of drug available was limited, tests were restricted to subjects whose preceding prepatent and latent periods showed no unusual prolongation.

Administration of drugs. For most therapeutic tests, treatment was begun at noon of the day after a patient had satisfied both of the following criteria :

1. Malarial parasites had been demonstrated in thick films of the peripheral blood for two consecutive days. The films were made as described above for prophylactic tests.

2. The rectal temperature had been $102^{\circ} \mathrm{F}$. or over.

The drugs were administered at four-hour intervals to obtain fairly constant concentrations in the body fluids, except that slowly excreted or slowly degraded drugs, such as $\mathrm{SN}-8617$, quinacrine, and chloroquine, were given less frequently. The usual period of drug administration was 14 days. The technique of drug administration was carried out as described above for the prophylactic tests.

Observations. Interim observations when patients were neither receiving drugs nor undergoing a malarial attack were made as follows:

Thick films of the peripheral blood were taken every other day and alternate films were studied 
for the presence of plasmodia. The films were studied as outlined above. Thus, the onset of peripheral parasitemia was localized within one day. Patients were admitted to the hospital either when they became febrile or when symptoms or heavy parasitemia made ambulatory status inadvisable. When parasitic latent periods exceeded six months, observations were made once a week.

During hospitalization for therapeutic studies, the same observations were made as are listed above under Prophylactic Tests.

The majority of drugs studied in therapeutic trials have been members of the 8-aminoquinoline group of compounds. For these drugs, the following additional tests were added to the above routine:

a. Leucocyte count every day and differential every fourth day, oftener when indicated.

$b$. Hemoglobin and methemoglobin determination every day, and on occasion, twice a day.

c. Electrocardiográm after treatment.

\section{COMMENT}

Using the technique outlined, it has been possible to develop a standardized procedure for the controlled clinical testing of new antimalarial agents. The actions of previously known drugs such as quinine, quinacrine, and pamaquin, have been documented quantitatively, and used as standards of comparison in the evaluation of new drugs. Thirty new compounds have been tested. ${ }^{5}$

\section{BIBLIOGRAPHY}

1. Craige, B., Jr., Alving, A. S., Jones, R., Jr., Whorton, C. M., Pullman, T. N., and Eichelberger, L., The Chesson strain of Plasmodium vivax malaria. II. Relationship between prepatent period, latent period and relapse rate. J. Infect. Dis., 1947, 80, 228.

2. Ehrman, F. C., Ellis, J. M., and Young, M. D., Plasmodium vivax, Chesson strain. Science, 1945, 101, 377.

3. Young, M. D., Ellis, J. M., and Stubbs, T. H., Studies on imported malarias. V. Transmission of foreign Plasmodium vivax by Anopheles quadrimaculatus. Am. J. Trop. Med., 1946, 26, 477.

4. Coatney, G. R., Cooper, W. C., Ruhe, D. S., and Young, M. D., Studies in human malaria. XVII. Trials of quinacrine, colchicine (SN 12,080) and quinine against Chesson strain vivax malaria. Am. J. Hyg. (to be published).

5. Coatney, G. R., Cooper, W. C., and Ruhe, D. S., Studies in human malaria. VI. Organization of a program for testing potential antimalarial drugs in prisoner-volunteers. Am. J. Hyg. (in press).

6. Earle, W. C., and Perez, M., Enumeration of parasites in the blood of malarial patients. J. Lab. \& Clin. Med., 1932, 17, 1124.

5 The authors wish to acknowledge the services of the civilian nursing and technical staff and the assistance of Civilian Public Service assignees. These investigations would not have been possible except for the enthusiastic cooperation of several hundred inmate volunteers at Stateville Penitentiary. The volunteers are too numerous to list individually. 